\title{
Cerebral Infarct Complicating Traumatic Pneumatocele: A Rare Sequela Following Blunt Chest Trauma
}

\author{
Emeka Kesieme ${ }^{1}$ Manfred Feldmann ${ }^{2}$ Katrin Welcker ${ }^{3}$ Albert Linder $^{3}$ Georgi Prisadov $^{3}$
}

${ }^{1}$ Irrua Specialist Teaching Hospital-Thoracic Surgery, Irrua, Nigeria

${ }^{2}$ Klinikum Bremen Ost-Neurology, Bremen, Germany

${ }^{3}$ Klinikum Bremen Ost-Thoracic Surgery, Bremen, Germany
Address for correspondence Dr. Katrin Welcker, FETCS, Department of Thoracic Surgery, Klinikum Bremen Ost, Zuricher Str. 40, Bremen, Germany (e-mail: katrin.welcker@klinikum-bremen-ost.de).

Thorac Cardiovasc Surg 2012;60(S2):e16-e18.
Abstract
Keywords
- cerebral infarct
- air embolism
- traumatic pneumatocele
- blunt chest trauma

Systemic air embolism is known to rarely complicate blunt chest trauma. However, cerebral infarction caused by air emboli possibly originating from a traumatic pneumatocele has not been previously reported. We report a case of a 46-year-old woman who sustained blunt chest trauma with multiple rib and clavicular fractures, hemothorax and a huge, tense traumatic pneumatocele. She subsequently developed clinical and radiologic features of cerebral infarction. The cerebral infarct is likely to be secondary to cerebral air embolism originating from a traumatic pneumatocele.

\section{Introduction}

Cerebral air embolism is a rare complication of blunt chest trauma with catastrophic consequence of infarction.

Following trauma, systemic air embolism has been known to follow intermittent positive pressure ventilation, coughing, and tension pneumothorax. ${ }^{1}$

Association between cerebral air embolism and traumatic pneumatocele has not been previously reported.

We present a 46-year-old woman who developed traumatic Pneumatocele following blunt chest trauma and subsequently developed features of cerebral infarction 4 days later.

\section{Case Report}

A 46-year-old woman fell down from a horse and landed on the left side of her chest. She sustained multiple left rib fractures (5th and 6th), left clavicular fracture, pulmonary contusion, and hemothorax. There was no history suggestive of head trauma.

Chest CT scan revealed pulmonary contusion, hemothorax, and a huge pneumatocele in the left lower lung zone ( - Fig. 1). She had closed tube thoracostomy drainage. (CTTD)
Four days posttrauma, she complained of severe headache, nausea, sensation of light flashes, and right visual field defect.

Brain CT scan and MRI revealed fresh infarcts mainly in the areas supplied by left cerebellar artery, left postero-occipital artery, and the deeper aspect of the medulla (-Fig. $\mathbf{2}$ ).

Further evaluation of the cardiovascular system revealed normal findings. Doppler ultrasonography of cerebral vessels, echocardiography, and Holter ECG were normal.

She had an anterolateral thoracotomy on account of suspected cerebral embolism arising from traumatic pneumatocele. Intraoperative findings included a huge pneumatocele under considerable tension and hemothorax. The pneumatocele was completely resected and postoperative period was uneventful (-Fig. 3).

A review of the patient 2 weeks after the discharge showed complete resolution of neurological symptoms.

\section{Discussion}

Traumatic pneumatocele is a rare complication of chest trauma. It is defined as an air filled cavitary lesion without epithelial lining developing in pulmonary parenchyma after blunt thoracic trauma. ${ }^{2}$
Dol http://dx.doi.org/ 10.1055/s-0032-1304549. ISSN 2194-7635. 


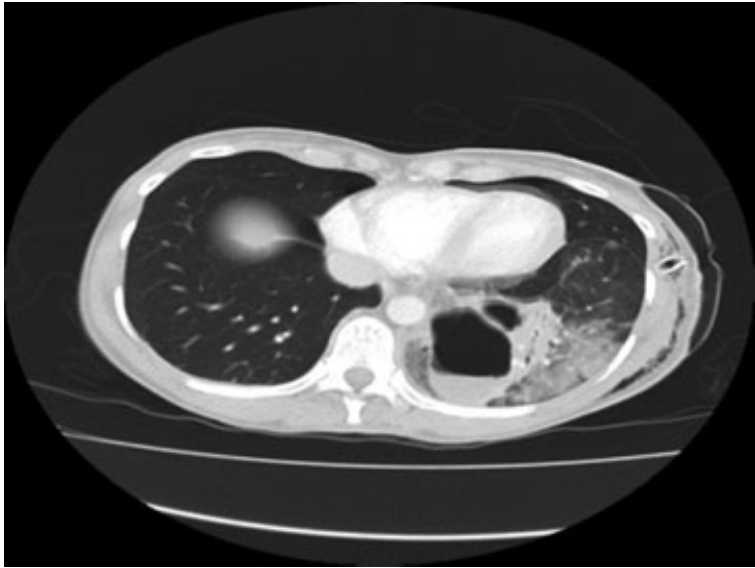

Figure $1 \mathrm{CT}$ of the chest showing traumatic pneumatocele, lung contusion, and left CTTD.

Unlike in the index patient, it is more common in children and young adults, as the formation of traumatic pneumatocele requires a violent impact directed on a pliable chest wall as seen in these age group, permitting transmission of kinetic energy to the underlying pulmonary parenchyma. ${ }^{3,4}$

Traumatic pneumatoceles result from pulmonary parenchymal laceration as the initial event that is followed by the normal elastic recoil of the surrounding lung with air trapping in the remaining cavity. ${ }^{2}$

The natural course of an uncomplicated traumatic pneumatocele is complete resolution and radiographic disappear-

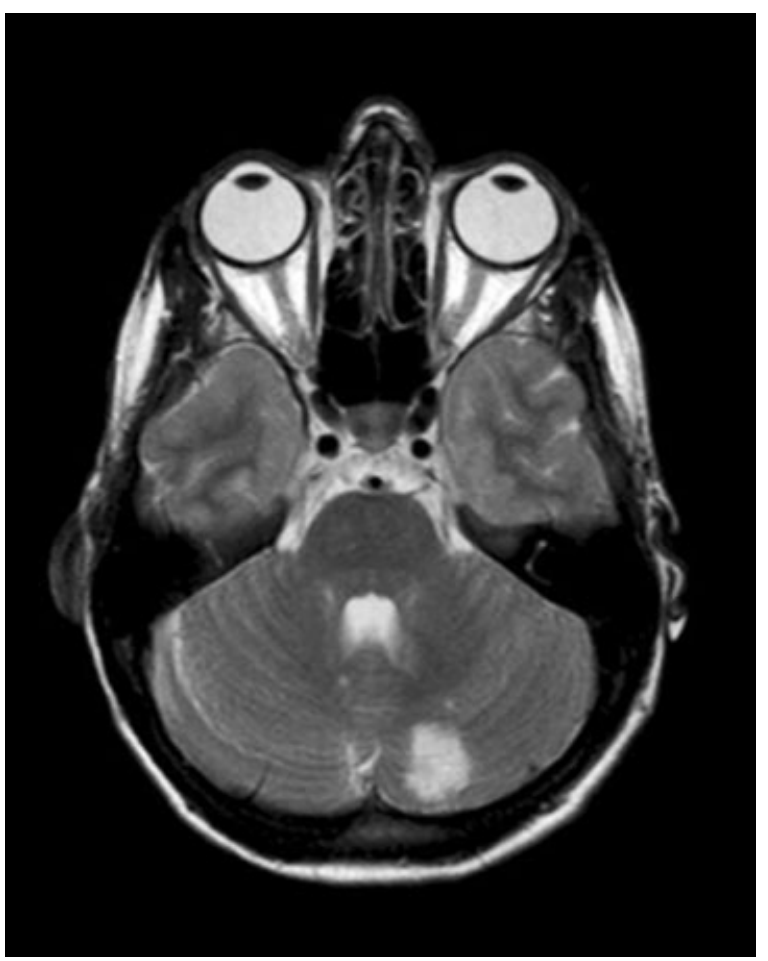

Figure 2 Brain MRI: infarction around the left cerebellar artery.

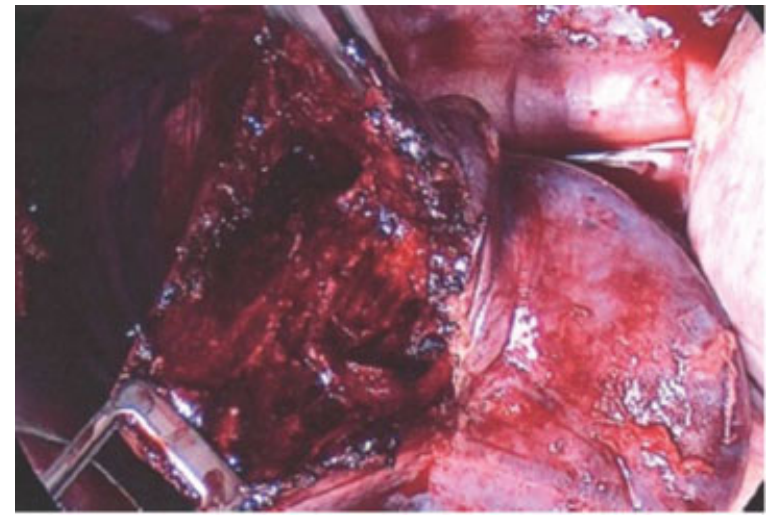

Figure 3 Cavity of pneumatocele opened.

ance; however complications associated with traumatic pneumatocele including secondary infection and hemoptysis have been reported. ${ }^{5}$

Although air embolism has been reported following the blunt chest trauma, there is no previous documentation of systemic air embolism or specifically cerebral air embolism arising from a traumatic pneumatocele.

Following trauma, air embolism result from creation of bronchopulmonary venous fistula. ${ }^{6}$ We hypothesize that air embolism resulted from the "tense" pneumatocele probably due to a pressure gradient between a pneumatocele under tension and an "open" pulmonary venous communication allowing the movement of air into the vein and the left side of the heart. This is likely since further cardiovascular evaluation on this patient revealed no abnormality and patient has been asymptomatic since surgical removal of the pneumatocele.

Injection of as little as 2 to $3 \mathrm{~mL}$ in the cerebral vasculature can be catastrophic with an undesirable outcome of distal infarction and acute inflammatory response. ${ }^{7}$ Cerebral embolism should be suspected in a traumatized patient, who had no history of head injury but developed neurological deficits subsequently. These neurological deficits include headache and visual field deficits in the index patient or coma, seizures, hemiparesis or paraesthesia.

Though fundoscopy was not performed in this patient, it may reveal air in the retinal vessels. Intracardiac air can be seen during echocardiography and air can be seen on Doppler ultrasonographic evaluation of cerebral vessels, but little amount of air is likely to be completely reabsorbed after many days of trauma.

Conservative treatment is recommended for uncomplicated traumatic pneumatocele since they resolve spontaneously, but complete resection is recommended for all known complicated cases. ${ }^{2}$ Systemic air embolism has a fatal outcome of $\sim 80 \%$ in blunt chest trauma ${ }^{8}$; hence surgical resection of pneumatocele through a thoracotomy is justified to remove the probable source of air embolism. 
e18 Cerebral Infarct Complicating Traumatic Pneumatocele

\section{References}

1 Ho AM. Is emergency thoracotomy always the most appropriate immediate intervention for systemic air embolism after lung trauma? Chest 1999;116(1):234-237

2 Melloni G, Cremona G, Ciriaco P, et al. Diagnosis and treatment of traumatic pulmonary pseudocysts. J Trauma 2003;54(4):737-743

3 Athanassiadi K, Gerazounis M, Kalantzi N, Kazakidis P, Fakou A, Kourousis D. Primary traumatic pulmonary pseudocysts: a rare entity. Eur J Cardiothorac Surg 2003;23(1):43-45

4 Crestanello JA, Samuels LE, Kaufman MS, Thomas MP, Talucci R. Posttraumatic pulmonary pseudocyst. J Trauma 1998;44(2): 401-403
5 Kocer B, Gulbahar G, Gunal N, Dural K, Sakinci U. Traumatic pulmonary pseuodocysts: two case reports. J Med Case Reports 2007; $1: 112$

6 Meier GH, Wood WJ, Symbas PN. Systemic air embolization from penetrating lung injury. Ann Thorac Surg 1979;27(2): $161-168$

7 Sinner WN. Complications of percutaneous transthoracic needle aspiration biopsy. Acta Radiol Diagn (Stockh) 1976;17(6): 813-828

8 Weber-Donat G, Donat N, Potet J, et al. Fatal systemic air embolism after blunt chest trauma: Postmortem computed-tomographic findings. Eur J Radiol Extra 2011;77(3):e67-e69 